\title{
Processus hydrogéochimiques et séparation d'hydrogrammes de crue sur un bassin versant en milieu soudano-tropical de socle au Bénin (Donga, haute vallée de l'Ouémé) Hydrogeochemical processes and hydrograph separation in the Donga catchment in Benin
}

\author{
Kamagaté Bamory, Séguis Luc, Goné Droh Lanciné, Favreau Guillaume et Koffi \\ Kouadio
}

Volume 21, numéro 3, 2008

URI : https://id.erudit.org/iderudit/018782ar

DOI : https://doi.org/10.7202/018782ar

Aller au sommaire du numéro

Éditeur(s)

Université du Québec - INRS-Eau, Terre et Environnement (INRS-ETE)

ISSN

0992-7158 (imprimé)

1718-8598 (numérique)

Découvrir la revue

Citer cet article

Bamory, K., Luc, S., Lanciné, G. D., Guillaume, F. \& Kouadio, K. (2008). Processus hydrogéochimiques et séparation d'hydrogrammes de crue sur un bassin versant en milieu soudano-tropical de socle au Bénin (Donga, haute vallée de l'Ouémé). Revue des sciences de l'eau / Journal of Water Science, 21(3), 363-372. https://doi.org/10.7202/018782ar
Résumé de l'article

Le projet international et pluridisciplinaire AMMA (Analyse Multidisciplinaire de la Mousson Africaine) a été initié en vue de mieux comprendre les variabilités climatiques de l'Afrique de l'Ouest et leur impact hydrologique. La haute vallée de l'Ouémé au Bénin $\left(10000 \mathrm{~km}^{2}\right)$ a été retenue et instrumentée (pluie, débit et nappe) depuis 1997. Le sous-bassin de la Donga $\left(586 \mathrm{~km}^{2}\right)$, cadre d'observations intensives depuis 2003, permet de préciser les processus majeurs et quantifier les termes du bilan hydrologique. Le travail vise à déterminer le fonctionnement hydrogéochimique du bassin de la Donga, et à identifier et quantifier les composantes majeures de l'écoulement de surface par traçage géochimique naturel.

Le caractère temporaire des eaux de surface, la très faible minéralisation des écoulements ainsi que l'asynchronisme entre le tarissement des rivières et la vidange de la nappe phréatique traduisent une origine superficielle des débits et un échange de flux négligeable entre cette nappe et le réseau hydrographique. L'écoulement à l'exutoire, limité à la saison des pluies, apparaît être essentiellement formé d'un flux rapide (ruissellement Hortonien et écoulement sur surfaces saturées) et d'un flux lent de subsurface (vidange de nappes perchées saisonnières), sans contribution significative de la nappe phréatique. En accord avec ce fonctionnement, une séparation géochimique de l'hydrogramme de trois crues a été réalisée.
Tous droits réservés ( $\odot$ Revue des sciences de l'eau, 2008
Ce document est protégé par la loi sur le droit d'auteur. L’utilisation des services d'Érudit (y compris la reproduction) est assujettie à sa politique d'utilisation que vous pouvez consulter en ligne.

https://apropos.erudit.org/fr/usagers/politique-dutilisation/ 


\title{
PROCESSUS HYDROGÉOCHIMIQUES ET SÉPARATION D'HYDROGRAMMES DE CRUE SUR UN BASSIN VERSANT EN MILIEU SOUDANO-TROPICAL DE SOCLE AU BÉNIN (DONGA, HAUTE VALLÉE DE L'OUÉMÉ)
}

\begin{abstract}
Hydrogeochemical processes and bydrograph separation in the Donga catchment in Benin
\end{abstract}

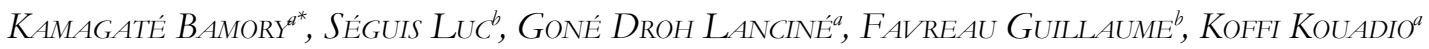

${ }^{a}$ Université d'Abobo-Adjamé, UFR des Sciences et Gestion de l'Environnement, Laboratoire de Géosciences et Environnement, 02 BP 801, Abidjan 02, Côte d'Ivoire

bUMR HydroSciences (IRD, CNRS, UMI, UMII), Université Montpellier-2, place Eugène-Bataillon, CC MSE, 34095 Montpellier cedex 5, France

Reçu le 21 septembre 2007, accepté le 27 février 2008

\section{RÉSUMÉ}

Le projet international et pluridisciplinaire AMMA (Analyse Multidisciplinaire de la Mousson Africaine) a été initié en vue de mieux comprendre les variabilités climatiques de l'Afrique de l'Ouest et leur impact hydrologique. La haute vallée de l'Ouémé au Bénin $\left(10000 \mathrm{~km}^{2}\right)$ a été retenue et instrumentée (pluie, débit et nappe) depuis 1997. Le sousbassin de la Donga $\left(586 \mathrm{~km}^{2}\right)$, cadre d'observations intensives depuis 2003, permet de préciser les processus majeurs et quantifier les termes du bilan hydrologique. Le travail vise à déterminer le fonctionnement hydrogéochimique du bassin de la Donga, et à identifier et quantifier les composantes majeures de l'écoulement de surface par traçage géochimique naturel.

Le caractère temporaire des eaux de surface, la très faible minéralisation des écoulements ainsi que l'asynchronisme entre le tarissement des rivières et la vidange de la nappe phréatique traduisent une origine superficielle des débits et un échange de flux négligeable entre cette nappe et le réseau hydrographique. L'écoulement à l'exutoire, limité à la saison des pluies, apparaît être essentiellement formé d'un flux rapide (ruissellement Hortonien et écoulement sur surfaces saturées) et d'un flux lent de subsurface (vidange de nappes perchées saisonnières), sans contribution significative de la nappe phréatique. En accord avec ce fonctionnement, une séparation géochimique de l'hydrogramme de trois crues a été réalisée.

Mots clés : Bénin, hydrochimie, hydrodynamique, rivière tropicale, séparation d'hydrogrammes.

\section{ABSTRACT}

The international and multidisciplinary AMMA project (African Monsoon Multidisciplinary Analysis) was designed

*Auteur pour correspondance :

Téléphone: (+225) 02200401

Télécopie : $\quad$ (+225) 20378118

Courriel : kambamory2@yahoo.fr 
to better understand the West African climatic variability and its hydrological impact. To attain this goal, the upper Oueme river $\left(10,000 \mathrm{~km}^{2}\right)$ was instrumented (rainfall, runoff and groundwater) in 1997. In 2003, the Donga subwatershed $\left(586 \mathrm{~km}^{2}\right)$ was selected in order to characterize the major processes and quantify components of the water budget. Specifically, the objective was to identify and quantify the major components of the surface flow using a ydrogeochemical approach.

The transient character of the surface water, the very low mineralization of the river as well as the asynchronism between the drying up of rivers and the water table height, suggest a shallow origin for the river flow and an unimportant exchange between that water table and the hydrographic network. River flow at the outlet, limited to the rainy season, appears to be essentially made of a rapid runoff (Hortonian runoff and flow over saturated soils) and a subsurface delayed flow without significant contribution of the water table. In agreement with this assumption, a hydrograph separation for three floods was achieved.

\section{Keywords: Benin, Hydrochemistry, Hydrodynamic, Tropical river, Hydrograph separation}

\section{INTRODUCTION}

Les régions ouest africaines, sous régime de mousson, sont l'objet d'une sécheresse sévère depuis le début des années 70 (LEBEL et VISCHEL, 2005; L'HÔTE et al., 2002). Ce phénomène est la conséquence d'une perturbation dela mousson africaine, première source des pluies de la région (LEBEL et VISCHEL, 2005). Le projet international et pluridisciplinaire AMMA (Analyse Multidisciplinaire de la Mousson Ouest Africaine) a été initié en vue de mieux comprendre cette perturbation. L'un des objectifs de ce programme, en relation avec notre travail, est la connaissance des bilans d'eau terrestre et l'impact des changements climatiques et des pressions anthropiques sur les ressources en eau.

Dans le cadre de l'Observatoire de Recherche en Environnement ORE AMMA-Catch (France, http://www.lthe. hmg.inpg.fr/catch), trois sites de méso-échelle, instrumentés de la zone soudanaise (Ouémé supérieur au centre-ouest du Bénin) à la zone désertique (Niamey au Niger) en passant par la zone sub-désertique (Gourma au Mali), font l'objet d'investigations renforcées. Le bassin versant de la Donga, situé dans la haute vallée de l'Ouémé (site le plus méridional du projet), a été sélectionné pour cette étude. D'une superficie de $586 \mathrm{~km}^{2}$, ce bassin représente une échelle intermédiaire entre les grands bassins régionaux et les bassins expérimentaux.
Cette échelle rend possible une caractérisation plus fine des processus hydrologiques, tout en permettant une intégration suffisamment représentative des variabilités spatio-temporelles naturelles.

Alors que l'écoulement des rivières en zone sahélienne est exclusivement formé d'écoulement de surface, du fait des sols peu couverts, des surfaces encroûtées (CASENAVE et VALENTIN, 1992) et des nappes relativement profondes (profondeur > 100 m; Favreau, 2000), l'écoulement en zone soudanienne se caractérise par une coexistence d'écoulement de surface et d'écoulement souterrain (CHEVALLIER, 1990; CHEVALLIER et PLANCHON, 1993). L'objectif de ce travail est de caractériser et évaluer les échanges de flux entre le réseau hydrographique et les nappes souterraines, ceci, en vue de mieux contraindre l'estimation du bilan hydrologique du bassin versant de la Donga sur deux années (2003 et 2004) ayant une pluviométrie et un écoulement contrastés. Il s'agit, d'une part, d'identifier les composantes principales de l'écoulement de surface et, d'autre part, de quantifier la contribution de chaque composante par une séparation géochimique d'hydrogrammes de crue.

\section{MATÉRIEL ET MÉTHODES}

\subsection{Milien d'étude}

Sous climat tropical de type soudano-guinéen à saisons contrastées, le bassin versant de la Donga se localise dans la partie supérieure du bassin de l'Ouémé, au centre-ouest du Bénin (Figure 1). Le réseau hydrographique est relativement dense et constitué de rivières d'ordre 2 ou 3 . Il présente un régime d'intermittence caractérisé par un assèchement total des rivières entre novembre et avril et une reprise des écoulements entre mai et octobre.

La pluviométrie moyenne annuelle est estimée à $1280 \mathrm{~mm}$ de 1950 à 1969 (avant la rupture pluviométrique) et de $1150 \mathrm{~mm}$ de 1970 à 2004 (après la rupture pluviométrique). Plus de $60 \%$ des pluies annuelles se concentrent entre les mois de juillet et septembre. L'évapotranspiration potentielle (ETP), estimée par la méthode de Penman-Monteith (FAO, 1998) est de l'ordre de $1390 \mathrm{~mm} \bullet \mathrm{an}^{-1}$ (KAMAGATÉ, 2006).

La topographie du bassin est vallonnée, avec des altitudes comprises entre $520 \mathrm{~m}$ à l'amont (ouest) et $340 \mathrm{~m}$ à l'exutoire (est) pour une pente moyenne de la rivière principale de $1,7 \mathrm{~m} \cdot \mathrm{km}^{-1}$. Ce relief laisse apparaitre une végétation constituée de savane arborée plus ou moins dense (à Isoberlinia $s p$. dominant) à couvert herbacé continu (Andropogon sp, Hypparhenia sp., Indigo fera; Harris, 2004). Cette végétation 


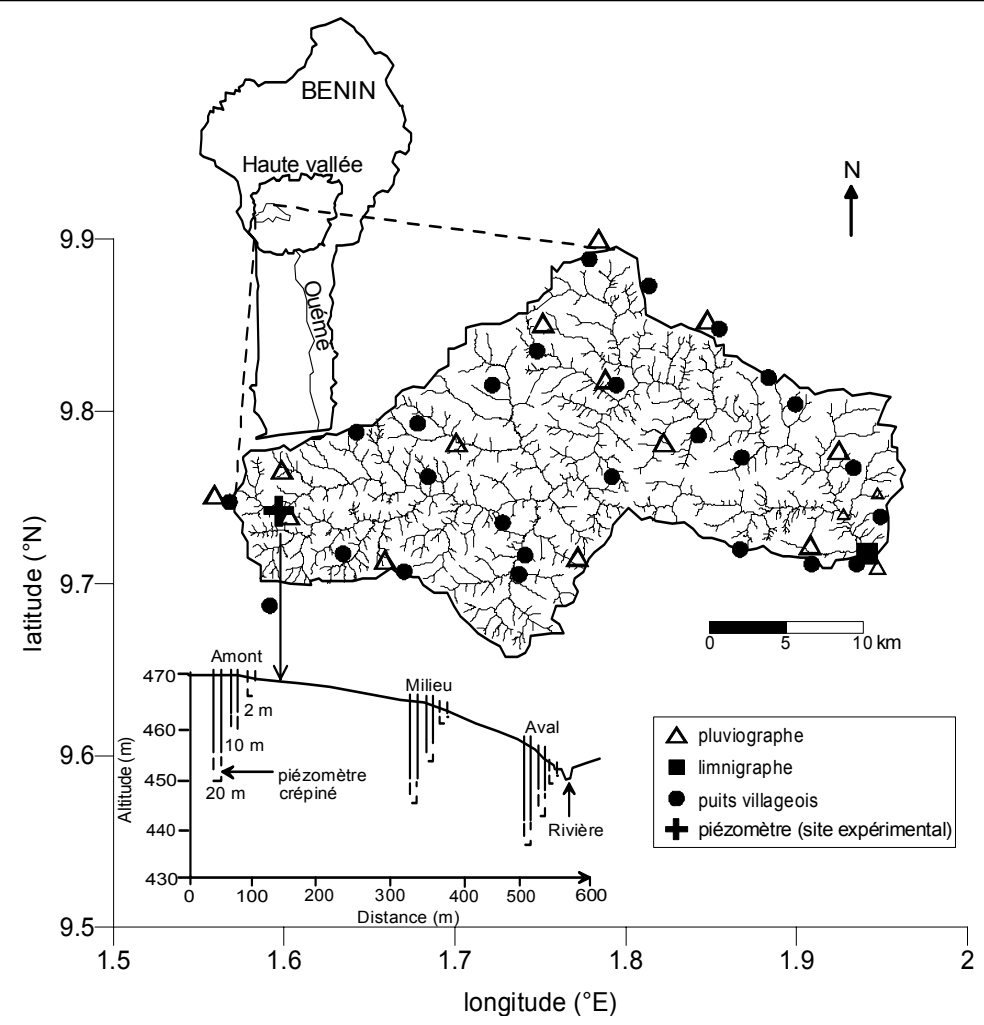

Figure1. Localisation du bassin versant de la Donga et dispositif de suivi hydrogéochimique.

Location of the Donga watershed and the hydrogeochemical survey network.

apparaît largement dégradée en une mosaïque de champs à vocation vivrière (mil, manioc, maïs, igname) ou de rente (coton, anacarde).

La couverture pédologique est majoritairement formée de sols ferrugineux tropicaux lessivés (FAURE et VOLKHOFF, 1998). Elle recouvre une zoned'altération (10à 20 md'épaisseur) à nappe pérenne (nappe phréatique), captée par les puits villageois (WUBDA, 2003). Cette altération, principalement silteuse et argileuse, présente une hétérogénéité importante en fonction de la nature de la roche et des teneurs en eau ou en argile selon la géophysique de subsurface (DESCLOITRES et al., 2003; ROBAIN et WUBDA, 2004; WUBDA, 2003). Les niveaux argilo-sableux, électriquement conducteurs, sont organisés en lentilles parfois affleurantes. Cette distribution suggère que des nappes perchées peuvent se mettre en place dans ces altérites, les formations argileuses constituant des niveaux imperméables. Du fait de la présence de ces niveaux imperméables, la nappe phréatique ne serait pas libre sur toute sa surface. Aussi, localement et plus en profondeur, des fractures du socle peuvent constituer un réservoir pérenne capté par les forages d'hydraulique villageoise généralement plus profonds que les puits villageois (WUBDA, 2003). Ce socle est essentiellement constitué de formations métamorphiques, datées du protérozoïque supérieur. Les granito-gneiss, les gneiss et les schistes sont les principales roches rencontrées (AFFATON, 1990; BRGM/OBM, 1984).

\subsection{Hydrogéochimie}

Sur l'ensemble du bassin, 16 pluviographes (Figure 1) permettent de discriminer la hauteur de pluie précipitée par cumuls de $0,5 \mathrm{~mm}$. Vingt-six puits villageois $(10 \mathrm{~m}$ de profondeur en moyenne) servent aux mesures des niveaux de la nappe phréatique. Parmi ces puits, 13 font à la fois l'objet de mesures tri-journalières (matin, midi et soir) et de mesures au pas de temps de 30 minutes. Les 13 autres sont suivis seulement trois fois par jour. À une échelle de plus fine investigation, neuf piézomètres (exempts de prélèvements humains, contrairement aux puits villageois) ont été crépinés à différentes profondeurs (2, 10 et $20 \mathrm{~m}$ de profondeur), au long d'un versant expérimental (Amont, Milieu et Aval) en 2004 (Figure 1). Ces piézomètres sont suivis trois fois par jour. Les forages d'hydraulique villageoise (profondeur $>20 \mathrm{~m}$ ) sont dotés d'un équipement inadapté aux mesures des hauteurs d'eau. Ils n'ont donc pas 
fait l'objet de mesures piézométriques. L'exutoire du bassin (Figure 1) bénéficie d'enregistrements de débits au pas de temps de 30 minutes, au moyen d'un limnigraphe. L'ensemble des piézomètres et l'échelle limnimétrique de l'exutoire ont été nivelés par GPS différentiel pour caractériser la distribution spatiale des potentiels hydrauliques.

La conductivité électrique (C.E.) de l'eau a été suivie à des pas de temps mensuels à trimestriels dans une vingtaine de puits et forages. Des mesures ponctuelles de C.E. ont également été réalisées lors d'une dizaine d'événements pluvieux, ainsi que sur les eaux de surface en période d'intercrue. En parallèle à ces mesures, un échantillonnage d'eau (C.E. et ions majeurs) à haute fréquence (pas de temps de $30 \mathrm{~min}$ ) et portant sur trois crues majeures (crue $\mathrm{A}$, crue $\mathrm{B}$ et crue $\mathrm{C}$ ) a été effectué à l'exutoire en 2003. L'ensemble des échantillons, filtrés in situ, a été analysé avec une précision de $5 \%$ par électrophorèse capillaire (cations et anions) au laboratoire HydroSciences de Montpellier (France).

Le croisement des informations hydrodynamiques et géochimiques recueillies devra permettre de dégager un schéma cohérent du fonctionnement hydrogéochimique du bassin versant de la Donga.

\subsection{Séparation géochimique d'hydrogrammes de crue}

Les rivières, exutoires naturels des différents réservoirs hydriques, jouent un rôle essentiel dans l'équilibre physicochimique et hydrodynamique du bassin versant (BRAUN et al., 2002). À un instant donné, les eaux recueillies à l'exutoire $\mathrm{du}$ bassin versant proviennent de plusieurs sources. Elles sont attribuées à la vidange de réservoirs spécifiques, situés à différentes profondeurs (BOEGLIN et TARDY, 1997). Le but de la séparation d'hydrogrammes, sur la base de la caractérisation hydrogéochimique des réservoirs hydriques, est de quantifier la contribution de chacun de ces réservoirs à la production des débits des rivières. Le modèle de séparation d'hydrogrammes considéré est régi par deux lois fondamentales de conservation de masse (BARIAC et al., 1995; JOERIN et al., 2002) :

1) la conservation de masse d'eau : l'écoulement total , mesuré à un instant $\mathrm{Q}(\mathrm{t})$ donné à l'exutoire, est la somme des écoulements $\mathrm{Q}_{\mathrm{k}}(\mathrm{t})$ (inconnues du modèle) induits par les réservoirs $\mathrm{k}$ identifiés (1).

$$
\mathrm{Q}(\mathrm{t})=\sum_{\mathrm{k}=1}^{\mathrm{n}} \mathrm{Q}_{\mathrm{k}}(\mathrm{t})
$$

où $\mathrm{n}$ représente le nombre de réservoirs $\mathrm{k}$.
2) la conservation de flux chimique : le flux $Q(t) \times C^{i}(t)$ d'un traceur chimique $\mathrm{i}$, calculé pour un instant $\mathrm{t}$ à l'exutoire, est la somme des flux $\mathrm{Q}_{\mathrm{k}}(\mathrm{t}) \times \mathrm{C}_{\mathrm{k}}^{\mathrm{i}}$ induits par les réservoirs $\mathrm{k}$ à cet instant (2).

$$
\mathrm{Q}(\mathrm{t}) \times \mathrm{C}^{\mathrm{i}}(\mathrm{t})=\sum_{\mathrm{i}=1}^{\mathrm{m}} \mathrm{Q}_{\mathrm{k}}(\mathrm{t}) \times \mathrm{C}_{\mathrm{k}}^{\mathrm{i}}
$$

où $\mathrm{m}=\mathrm{n}-1$ représente le nombre de traceurs chimiques $\mathrm{i}, \mathrm{C}^{\mathrm{i}}(\mathrm{t})$ la concentration du traceur i mesurée à l'exutoire à l'instant $t, C_{k}^{i}$ la concentration du traceur i (supposée constante) mesurée dans le réservoir k.

La résolution combinée des équations (1) et (2) ramène à la résolution (par la méthode des déterminants) de $\mathrm{n}$ d'équations à $n$ inconnues (3).

$$
\left(\begin{array}{ccccc}
1 & 1 & 1 & 1 & 1 \\
\mathrm{C}_{1}^{1} & \cdot & \cdot & \cdot & \mathrm{C}_{\mathrm{n}}^{1} \\
\cdot & \cdot & \cdot & \cdot & \cdot \\
\cdot & \cdot & \cdot & \cdot & \cdot \\
\cdot & \cdot & \cdot & \cdot & \cdot \\
\mathrm{C}_{1}^{\mathrm{m}} & \cdot & \cdot & \cdot & \mathrm{C}_{\mathrm{n}}^{\mathrm{m}}
\end{array}\right) *\left(\begin{array}{c}
\mathrm{Q}_{1} \\
\mathrm{Q}_{2} \\
\cdot \\
\cdot \\
\cdot \\
\mathrm{Q}_{\mathrm{n}}
\end{array}\right)=\left(\begin{array}{c}
\mathrm{Q} \\
\mathrm{Q}^{*} \mathrm{C}^{1} \\
\cdot \\
\cdot \\
\cdot \\
\mathrm{Q}^{*} \mathrm{C}^{\mathrm{m}}
\end{array}\right)
$$

Le premier terme de l'équation est formé par le produit de la matrice des constantes du modèle (concentrations $C_{k}^{i}$ des traceurs $\mathrm{i} \in[1, \mathrm{~m}]$ mesurées dans les réservoirs $\mathrm{k} \in[1, \mathrm{n}])$ par la matrice des inconnues (contribution $\mathrm{Q}_{\mathrm{k}}$ des réservoirs $\mathrm{k}$ ) et le deuxième terme par la matrice des flux $Q * C^{i}$ calculés pour les traceurs i à l'exutoire.

Ces deux lois de conservation de masse signifient que la variation de la charge hydraulique dans les rivières s'accompagne toujours d'une variation de la composition chimique de l'eau (APPELO et POSTMA, 1999). Cette approche nécessite la prise en compte d'un certain nombre d'hypothèses :

1) la concentration du traceur chimique dans l'épisode pluvieux considéré est significativement différente de celle de la rivière avant l'événement pluvieux (CROUZET et al., 1970). Cette hypothèse est vérifiée au regard de la grande différence chimique entre les eaux de pluie et les débits d'intercrue (KAMAGATÉ, 2006);

2) la concentration du traceur chimique dans l'eau de pluie ne présente pas de variations spatio-temporelles (SKLASH et FARVOLDEN, 1982). De façon générale, la composition chimique des pluies tend à se stabiliser sur une surface donnée au cours de l'averse (DANSGAARD, 1964; KENDALL et MCDONNELL, 1993); 
3) la composition chimique des eaux souterraines reste constante au cours des événements de crue (SKLASH et FARVOLDEN, 1982). Cette hypothèse reste discutable du fait de l'influence probable des eaux de recharge au cours de l'averse. Mais ce phénomène est rarement mis en évidence dans la littérature (HOOPER et SHOEMARKER, 1986);

4) La composition chimique des rivières en période d'écoulement de base est significativement proche de celle des eaux souterraines (SKLASH et FARVOLDEN, 1982). En période d'intercrue, il est évident que la production de débit ne peut qu'être le fait des eaux souterraines. L'analyse qui va suivre devra permettre d'identifier les aquiferes responsables de ces apports.

\section{RÉSULTATS ET DISCUSSION}

\subsection{Processus hydrogéochimiques}

\subsubsection{Caractéristiques hydrogéochimiques}

La pluviométrie annuelle (Figure 2A) présente une diminution importante de l'ordre de $24 \%$ entre 2003 $(1459 \mathrm{~mm})$ et $2004(1113 \mathrm{~mm})$. Cette différence est particulièrement marquée dans les écoulements (Figure 2B) : $429 \mathrm{~mm}$ en 2003 et $162 \mathrm{~mm}$ en 2004, soit une baisse de l'ordre de $60 \%$. Le coefficient d'écoulement annuel s'évalue à $29 \%$ en 2003 et $15 \%$ en 2004 . La durée de l'écoulement de la rivière, tributaire de l'occurrence de pluies, apparaît plus courte en 2004 qu'en 2003 (Figures 2A et 2B). Aussi, la rivière tarit-elle dès que la pluie s'arrête alors que la nappe phréatique continue de se vidanger (Figures $2 \mathrm{~B}$ et $2 \mathrm{C}$ ). De même que les écoulements de surface, la hausse piézométrique démarre environ un mois après les premières pluies et le pic est atteint trois à quatre mois plus tard. La décrue piézométrique s'amorce dès la fin de l'occurrence des précipitations régulières (Figure 2C). Les fluctuations piézométriques montrent, en comparaison interannuelle, une différence moindre que celle des débits : l'amplitude piézométrique moyenne annuelle varie de $7,4 \pm 2,8 \mathrm{~m}$ en 2003 à $5,3 \pm 2,3 \mathrm{~m}$ en 2004 (Figure 2C). Sur le site expérimental plus finement équipé, les piézomètres de 10 et $20 \mathrm{~m}$ de profondeur montrent une piézométrie semblable. L'amplitude piézométrique apparaît plus faible que celle enregistrée pour les puits villageois, plus exposés à un rabattement local du fait des puisages domestiques. Les piézomètres de $2 \mathrm{~m}$ de profondeur captent un niveau d'eau plus près de la surface du sol (environ $1 \mathrm{~m}$ au-dessus des niveaux lus dans les piézomètres de 10 et $20 \mathrm{~m}$ de profondeur) et ayant un caractère temporaire (Figure 3).

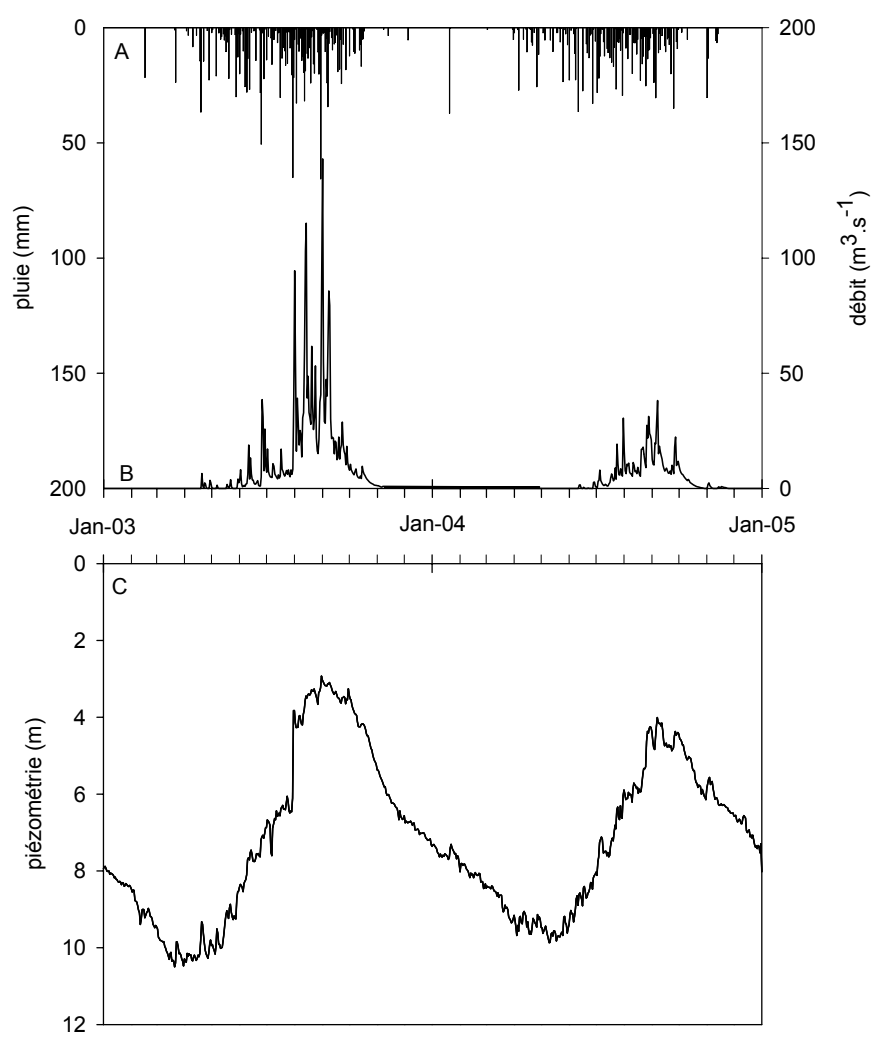

Figure 2. (A) Pluies journalières, (B) débits journaliers à l'exutoire du bassin, et $(C)$ profondeur moyenne journalière de la nappe phréatique pour les années 2003 et 2004.

(A) Daily rainfall, (B) daily runoff at the watershed outlet, and $(C)$ mean daily water table depth for the years 2003 and 2004.

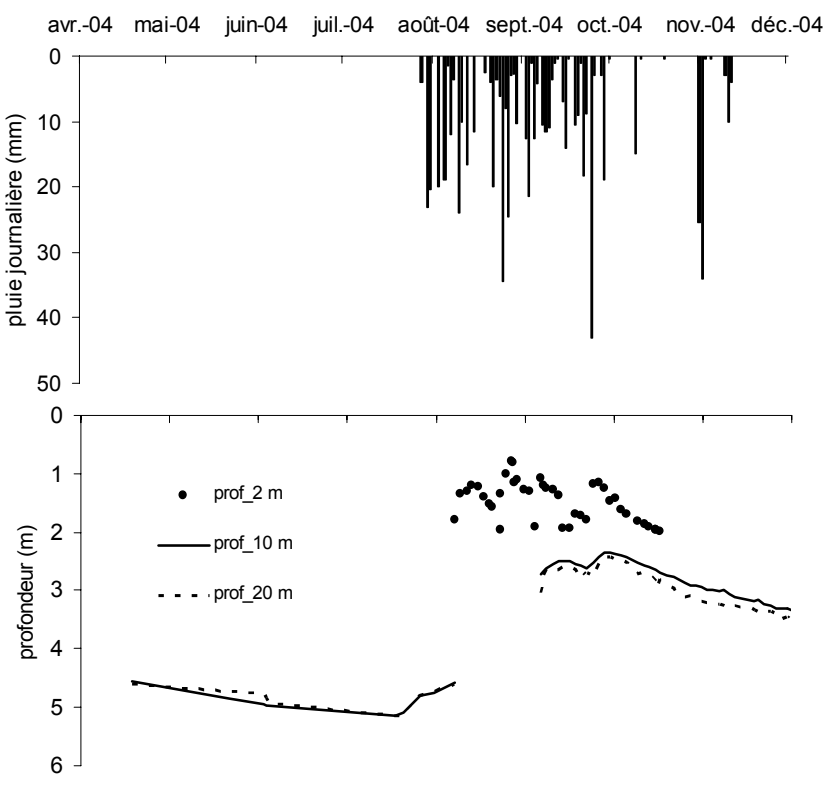

Figure 3. Profondeur de la nappe phréatique mesurée trois fois par jour dans les piézomètres de la station expérimentale avale $(2,10$ et $20 \mathrm{~m}$ de profondeur) et pluviométrie journalière mesurée à la station la plus proche en 2004.

Water table depth measured three times per day in the downgradient experimental piezometers (2,10 and $20 \mathrm{~m}$ of depth) and daily rainfall measured at the nearest station in 2004. 
La conductivité électrique des pluies est de l'ordre de $10 \pm 2 \mu \mathrm{S} \bullet \mathrm{cm}^{-1}$. Elle est de $65 \pm 6 \mu \mathrm{S} \bullet \mathrm{cm}^{-1}$ en moyenne à l'exutoire en période hors crue (Tableau 1, Figure 4) alors qu'en période de crues, elle baisse fortement et avoisine celle des pluies (Figure 4). La C.E. de la nappe phréatique (eaux de puits) dans les altérites montre des valeurs nettement supérieures avec une moyenne de $155 \pm 70 \mu \mathrm{S} \bullet \mathrm{cm}^{-1}$. Plus en profondeur, les eaux du socle fissuré (eaux de forage) montrent des valeurs moyennes encore plus élevées $\left(288 \pm 80 \mu \mathrm{S} \bullet \mathrm{cm}^{-1}\right)$. Sur le site de plus fine investigation, les suivis hydrochimiques permettent de préciser ces observations. La nappe captée par les piézomètres de 10 et $20 \mathrm{~m}$ de profondeur présente des valeurs de C.E. respectivement de $204 \pm 15$ et $254 \pm 30 \mu \mathrm{S} \bullet \mathrm{cm}^{-1}$ (Tableau 1) alors que celle captée par les piézomètres de $2 \mathrm{~m}$ de profondeur montre des C.E. fluctuant autour d'une moyenne de $75 \pm 3 \mu \mathrm{S} \bullet \mathrm{cm}^{-1}$, proche de celle des rivières en période d'intercrue.

\subsubsection{Relation nappe-réseau hydrographique}

À l'échelle des versants, l'altitude de la surface de la nappe phréatique, mesurée à partir des 26 puits villageois, est toujours

Tableau 1. Conductivité électrique moyenne calculée pour chaque système hydrique considéré.

Table 1. Average electrical conductivity calculated for each considered water system.

\begin{tabular}{lc}
\hline \multicolumn{1}{c}{ Système hydrique } & Conductivité électrique $\left(\boldsymbol{\mu} \mathbf{S}^{\bullet} \mathbf{c m}^{-1}\right)$ \\
\hline Pluie & $10 \pm 2$ \\
Eaux de surface & $65 \pm 6$ \\
(exutoire, hors crue) & $155 \pm 70$ \\
Altérite (puits) & $288 \pm 80$ \\
Socle (forage) & $75 \pm 5$ \\
Piézomètre $2 \mathrm{~m}$ & $204 \pm 15$ \\
Piézomètre $10 \mathrm{~m}$ & $254 \pm 30$ \\
Piézomètre $20 \mathrm{~m}$ & \\
\hline
\end{tabular}

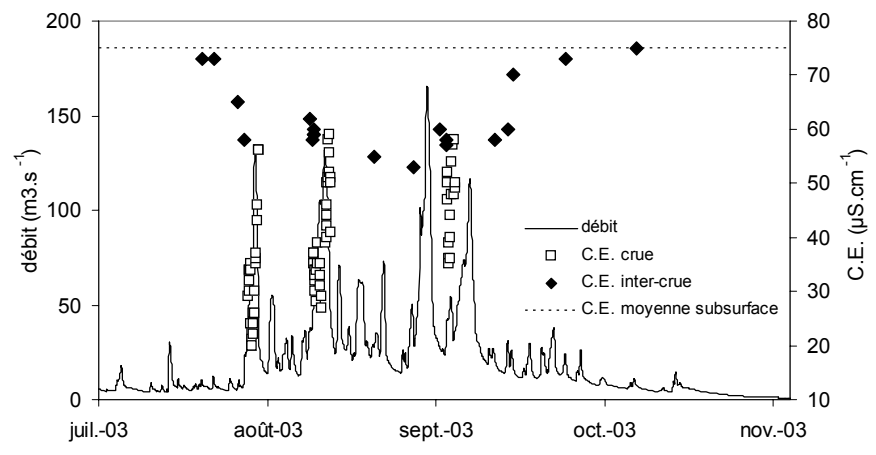

Figure 4. Conductivité électrique (C.E.) mesurée à l'exutoire en périodes de crues et intercrues pour l'année 2003.

Outlet measured electrical conductivity (C.E.) during flood and inter-flood periods in 2003. supérieure à celle du lit de la rivière, même en saison sèche (Figure 5). Ceci traduirait une alimentation des rivières par cette nappe indépendamment de la saison (KAMAGATÉ, 2003). Cependant, les cours d'eau s'assèchent totalement en fin de saison pluvieuse, sans débit de base, alors que la nappe phréatique continue de se vidanger. Le réseau hydrographique ne constituerait donc pas le principal exutoire de la nappe phréatique. Outre les prélèvements humains, la vidange de cette nappe serait essentiellement le fait des transferts verticaux par évapotranspiration. D'un point de vue géologique, une perte d'eau dans le socle fissuré via des brèches tectoniques à forte porosité ne serait pas totalement à exclure car quatre générations de fractures caractérisées par des couloirs mylonitiques à brèches tectoniques ont déjà été mises en évidence (AFFATON, 1990).

Le caractère temporaire des eaux de surface, le coefficient d'écoulement annuel relativement faible ainsi que la très faible minéralisation des eaux de la rivière suggèrent une origine superficielle des écoulements. Sur le site d'investigation renforcée, la nappe saisonnière à faible minéralisation, captée à $2 \mathrm{~m}$ de profondeur, a été identifiée comme une nappe perchée temporaire (KAMAGATÉ, 2006). La saisonnalitéde cette nappe tout comme l'écoulement des rivières, l'émergence de surfaces saturées en de multiples points du bassin, la forte hétérogénéité de la couche d'altérite avec présence de niveaux argilo-sableux électriquement conducteurs confortent l'existence de ces nappes perchées. Selon ce schéma, le débit à l'exutoire pourrait être considéré comme un mélange de flux rapides (ruissellement Hortonien et écoulement sur surfaces saturées) et de flux lents (vidange de la nappe perchée saisonnière), sans contribution significative de la nappe phréatique dans les altérites et le socle fissuré à l'écoulement.

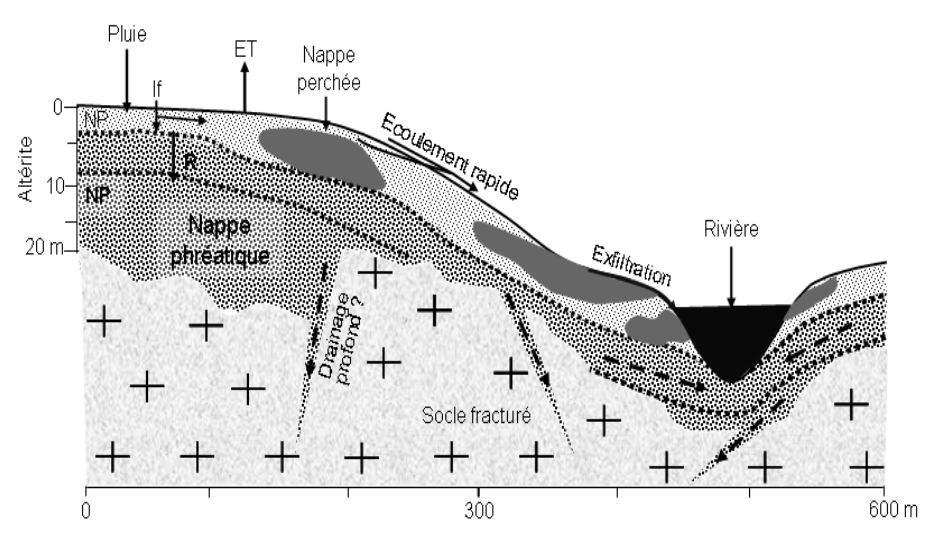

Figure 5. Schéma conceptuel des flux hydriques à l'échelle d'un versant, d'après les observations hydrologiques, géochimiques et géophysiques, ET : évapotranspiration, NP : niveau piézométrique saisonnier minimum et maximum de la nappe phréatique, If : infiltration et $\mathrm{R}$ : recharge.

Conceptual cross-section of the water flux at a billslope scale, according to the hydrological, geochemical, and geophysical observations, ET: evapotranspiration, NP: minimum and maximum seasonal levels of the water table (dotted lines), If: infiltration and $R$ : recharge. 
Un schéma conceptuel réalisé à l'échelle d'un versant, d'après les observations hydrologiques et géochimiques précédentes, appuyées par la géophysique de surface (ROBAIN et WUBDA, 2004), permet de préciser les flux hydriques (Figure 5) et d'appliquer l'équation (3) au bassin de la Donga (4) : un système de deux équations à deux inconnues; pour deux composantes, un seul traceur chimique est requis

$$
\left(\begin{array}{cc}
1 & 1 \\
C_{\mathrm{r}}^{1} & \mathrm{C}_{\mathrm{l}}^{1}
\end{array}\right) *\left(\begin{array}{c}
\mathrm{Q}_{\mathrm{r}} \\
\mathrm{Q}_{\mathrm{l}}
\end{array}\right)=\left(\begin{array}{c}
\mathrm{Q} \\
\mathrm{Q}^{*} \mathrm{C}^{1}
\end{array}\right)
$$

où $C_{r}^{1}$ représente la concentration du traceur choisi dans l'écoulement rapide (constante du modèle), $\mathrm{C}_{1}^{1}$ la concentration du traceur choisi dans l'écoulement lent (constante du modèle), $\mathrm{C}^{1}$ la concentration du traceur choisi à l'exutoire (mesurée au pas de temps de $30 \mathrm{~min}$ ), Q le débit total à l'exutoire (mesuré au pas de temps de $30 \mathrm{~min}$ ), $\mathrm{Q}_{\mathrm{r}}$ la part de l'écoulement rapide (inconnue du modèle) et $\mathrm{Q}_{1}$ la part de l'écoulement lent (inconnue du modèle).

\subsection{Séparation d'hydrogrammes de crue}

Les traceurs chimiques $\mathrm{Ca}$ et $\mathrm{Na}$ ont été choisis pour la séparation d'hydrogrammes. Le choix de ces deux traceurs relève d'une part du fait qu'ils sont des produits d'altération de roches, donc de bons traceurs d'origine d'eau et, d'autre part, du fait qu'ils présentent la même évolution que la C.E. lors des crues. La séparation d'hydrogrammes ne concerne que les crues car en période d'intercrue, seule la composante souterraine, représentée ici par les flux venant des nappes perchées, contribue à l'écoulement (BARIAC et al., 1995; GREMILLON et al., 2000; TARDY et al., 2004). La mise en œuvre du modèle de mélange nécessite la connaissance des valeurs numériques des constantes (concentration moyenne de $\mathrm{Ca}$ et $\mathrm{Na}$ ), relativement à l'écoulement rapide et à l'écoulement lent (pôles extrêmes d'influence) qui contribuent en proportion variable à la production de l'écoulement des rivières :

a) la minéralisation de l'eau de rivière se rapproche fortement de celle de l'eau de pluie durant les pics de crue, ce qui peut justifier l'assimilation des pluies au pôle écoulement rapide; $\mathrm{C}_{\mathrm{r}}^{1}=0,16 \mathrm{mg} \bullet \mathrm{L}^{-1}$ pour Ca et $0,09 \mathrm{mg} \bullet \mathrm{L}^{-1}$ pour $\mathrm{Na}$;

b) la minéralisation de l'eau de rivière se rapproche fortement de celle des nappes perchées en période d'intercrue; $\mathrm{C}_{1}^{1}=4,00 \mathrm{mg} \bullet \mathrm{L}^{-1}$ pour $\mathrm{Ca}$ et $1,14 \mathrm{mg} \cdot \mathrm{L}^{-1}$ pour $\mathrm{Na}$.

L'hydrogramme total de la crue A, échantillonnée en début de saison humide, est relatif à une crue unique en forme de cloche (Figure 6A). Il varie de $50 \mathrm{~m}^{3} \bullet \mathrm{s}^{-1}$ en début d'événement à $135 \mathrm{~m}^{3} \bullet \mathrm{s}^{-1}$ au pic de crue. À une chute des concentrations de
$\mathrm{Ca}$ et $\mathrm{Na}$ au début de la crue, suit un recouvrement progressif des valeurs à la décrue. Ces concentrations deviennent encore plus fortes lorsque le débit total devient inférieur à celui de début de crue. Sur la base des variations de concentration des traceurs, la séparation de l'hydrogramme indique une gamme de variations des flux lents (nappes perchées) de $20 \mathrm{~m}^{3} \bullet \mathrm{s}^{-1}$ à $50 \mathrm{~m}^{3} \bullet \mathrm{s}^{-1}$ (soit une contribution maximale de $37 \%$ au débit total) relativement à $\mathrm{Ca}$ (Figure 6A1). Cette gamme est de $17 \mathrm{~m}^{3} \bullet \mathrm{s}^{-1}$ à $30 \mathrm{~m}^{3} \bullet \mathrm{s}^{-1}$ (soit une contribution maximale de $22 \%$ au débit total) relativement à $\mathrm{Na}$ (Figure 6A2).

Contrairement à l'hydrogramme de la crue A, l'hydrogramme de la crue $\mathrm{B}$, échantillonnée en milieu de saison, est symptomatique de trois crues superposées (Figure 6B). Il débute à $60 \mathrm{~m}^{3} \bullet \mathrm{s}^{-1}$ et culmine au pic de crue à environ $120 \mathrm{~m}^{3} \bullet \mathrm{s}^{-1}$. Tout comme la première crue, les concentrations de $\mathrm{Ca}$ et $\mathrm{Na}$ à la décrue avoisinent celles de début de crue et deviennent supérieures par la suite. Les débits simulés pour les flux lents sont fluctuants, mais la valeur maximale est de l'ordre de $50 \mathrm{~m}^{3} \bullet \mathrm{s}^{-1}$ (soit $42 \%$ du débit total) pour le traceur Ca (Figure 6B1) et de $40 \mathrm{~m}^{3} \bullet \mathrm{s}^{-1}$ (soit $33 \%$ du débit total) pour le traceur $\mathrm{Na}$ (Figure 6B2).

L'hydrogramme total de la crue C, scrutée en fin de saison humide, est composé de deux événements, mais tronquée du fait de l'absence du début de l'événement global (Figure 6C). Les fluctuations des flux lents apparaissent identiques à celles du débit total avec un maximum de l'ordre de $30 \mathrm{~m}^{3} \bullet \mathrm{s}^{-1}$ (soit $58 \%$ du débit total) à partir du traceur Ca (Figure 6C1) et de $20 \mathrm{~m}^{3} \mathrm{~s}^{-1}$ (soit $39 \%$ du débit total à partir du traceur $\mathrm{Na}$ (Figure 6C2).

La contribution de l'écoulement rapide est calculée à partir de la différence entre l'écoulement total et l'écoulement lent pour chacune des trois crues. Sur la base des variations instantanées des débits simulés, la contribution totale de l'écoulement rapide et de l'écoulement lent a été calculée pour les trois crues (Tableau 2). Le bilan global se discrimine logiquement entre une composante majoritaire, l'écoulement rapide et une composante minoritaire, l'écoulement lent. Quoique toujours inférieure à l'écoulement rapide, la part de l'écoulement lent des nappes perchées apparaît croissante au fur et à mesure que la saison humide s'installe du fait de l'augmentation progressive de la pluie efficace.

Au regard de la différence sensible entre les résultats obtenus avec le traceur $\mathrm{Ca}$ et ceux obtenus avec le traceur $\mathrm{Na}$, les contributions simulées doivent être considérées avec circonspection et seules les gammes de variations et les processus majeurs peuvent être retenus. Ceci serait lié à la différence de solubilité, de valence et de rayon ionique entre ces deux traceurs, et probablement aux réactions d'adsorption et précipitation. 

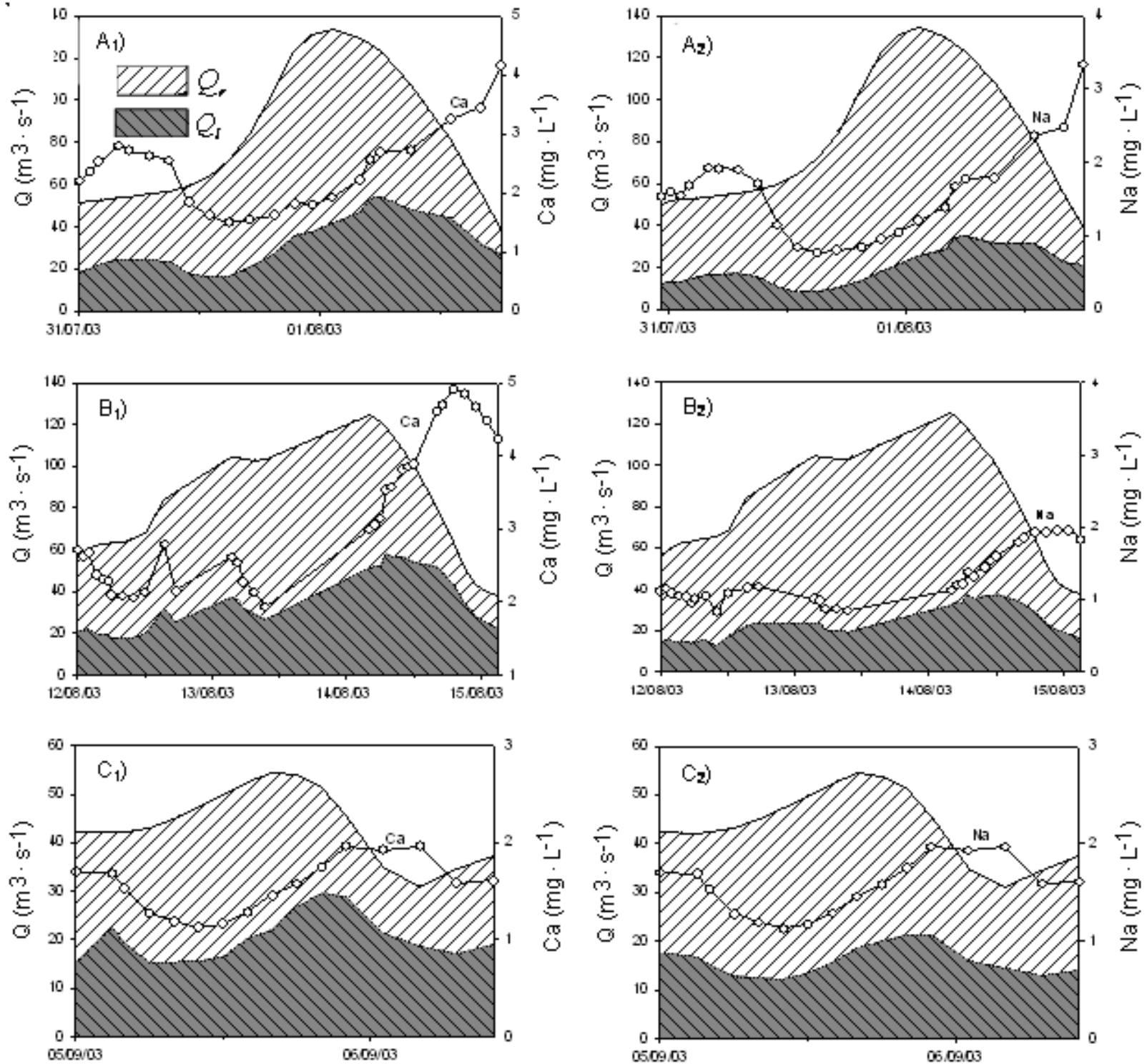

Figure 6. Séparation de l'hydrogramme des crues $A, B$ et $C$ en écoulement rapide $\left(Q_{r}\right)$ et écoulement lent $\left(Q_{l}\right)$ à l'exutoire du bassin de la Donga à partir des traceurs chimiques $\mathrm{Ca}(1)$ et $\mathrm{Na}(2)$.

Flood $A, B$ and $C$ hydrograph separation in rapid runoff $\left(\mathrm{Q}_{\mathrm{r}}\right)$ and delayed flow $\left(\mathrm{Q}_{1}\right)$ at the watershed outlet by mean of the chemical tracers $\mathrm{Ca}$ (1) and $\mathrm{Na}$ (2).

Tableau 2. Volume total drainé (V) par les crues A, B et C et contributions (\%) de l'écoulement rapide $\left(Q_{r}\right)$ et de l'écoulement lent $\left(Q_{l}\right)$ à l'écoulement total, obtenus après séparation d'hydrogrammes au moyen des traceurs chimiques $\mathrm{Ca}$ et $\mathrm{Na}$.

Table 2. Total drained volume $(V)$ by the floods $A, B$ and $C$ and rapid runoff $\left(Q_{r}\right)$ and delayed flow $\left(Q_{l}\right)$ contribution (\%) to the stream flow, obtained after the hydrographs separation by means of the chemical tracers Ca and Na.

\begin{tabular}{|c|c|c|c|c|c|c|}
\hline & \multicolumn{2}{|c|}{ Crue A } & \multicolumn{2}{|c|}{ Crue B } & \multicolumn{2}{|c|}{ Crue C } \\
\hline & \multicolumn{2}{|c|}{$\mathrm{V}_{\mathrm{t}}=1639000 \mathrm{~m}^{3}$} & \multicolumn{2}{|c|}{$\mathrm{V}_{\mathrm{t}}=3025000 \mathrm{~m}^{3}$} & \multicolumn{2}{|c|}{$\mathrm{V}_{\mathrm{t}}=549000 \mathrm{~m}^{3}$} \\
\hline Traceurs & $Q_{l}(\%)$ & $Q_{r}(\mathbf{0})$ & $Q_{l}(\mathbf{0})$ & $Q_{r}(\mathbf{0})$ & $Q_{l}(\mathbf{0})$ & $Q_{r}(\mathbf{\%})$ \\
\hline $\mathrm{Ca}$ & $37 \pm 12$ & 63 & $44 \pm 16$ & 56 & $46 \pm 11$ & 54 \\
\hline $\mathrm{Na}$ & $25 \pm 10$ & 75 & $32 \pm 12$ & 68 & $36 \pm 07$ & 64 \\
\hline
\end{tabular}




\section{CONCLUSION}

Le croisement des informations hydrodynamiques, géochimiques et géophysiques a permis de montrer que les eaux de surface sont majoritairement d'origine superficielle lors des crues. En période d'intercrue, elles sont essentiellement le fait de la vidange de nappes perchées saisonnières, sans contribution significative des nappes pérennes des altérites et du socle. La contribution de ces nappes perchées apparaît croissante avec la saison pluvieuse.

Ces résultats constituent une étape préliminaire dans l'étude hydrologique du bassin versant de la Donga. Un suivi à plus long terme des écoulements de surface (crue et intercrue) permettrait notamment de préciser l'importance respective des écoulements rapide et lent dans la variabilité interannuelle des débits des cours d'eau. Dans une perspective plus large, cette étude représente un début à une meilleure connaissance de l'impact du déboisement et du changement d'occupation des sols sur l'hydrologie de la zone. En effet, un défrichement continu et d'ampleur importante provoquerait une augmentation conséquente du coefficient de ruissellement du bassin (CASENAVE et VALENTIN, 1992). L'écart entre la contribution de l'écoulement rapide et celle de l'écoulement lent deviendrait de plus en plus important et la recharge des aquifères de plus en plus faible. Le défrichement pourrait également induire une diminution de l'évapotranspiration réelle (ETR) du fait d'un couvert végétal de moins en moins dense. Ceci met en exergue la nécessité de connaître l'ETR, jusque là inconnue, et son impact sur le bilan hydrologique du bassin.

\section{REMERCIEMENTS}

Nos remerciements vont à l'endroit de tous les chercheurs et techniciens de l'IRD au Bénin pour l'acquisition et la mise en forme des données. La Direction de l'Hydraulique de Djougou (MMEH, Bénin) est à remercier pour avoir autorisé l'accès aux infrastructures hydrauliques du bassin. L'accueil et la participation des habitants à certains suivis sont également remerciés.

\section{RÉFÉRENCES BIBLIOGRAPHIES}

AFFATON P. (1990). Le Bassin des Voltas (Afrique de l'Ouest): une marge passive, d'âge protérozoïque supérieur, tectonisée au panafricain $(600 \pm 50 \mathrm{Ma})$. Thèse de doctorat de Géologie, Université Aix-Marseille, France, 499 p.
APPELO C.A.J. et D. POSTMA (1999). Geochemistry, groundwater and pollution. Balkema, Rotterdam, Hollande, 536 p.

BARIAC T., A. MILLET, B. LADOUCHE, R. MATHIEU, C. GRIMALDI, M. GRIMALDI, M. SARRAZIN, P. HUBERT, H. MOLICOVA, L. BRUCKLER, V. VALLES, P. BERTUZZI, B. BES, J.C. GAUDU, J. HOROYAN, J. BOULEGUE, F. JUNG, Y. BRUNET, J.M. BONNEFOND, R. TOURNEBIZE et A. GRANIER (1995). Décomposition géochimique de l'hydrogramme de crue sur un petit bassin versant Guyanais (Piste Saint-Elie, dispositif ECEREX, Orstom-CTFT, Guyane Française). IASH, 238, 249-269.

BOEGLIN J.L. et Y. TARDY (1997). Érosion chimique et mécanique sur le bassin amont du Niger (Guinée, Mali). Découpage en quatre écoulements. CR Geosci., 325, 125-131.

BRAUN J.J., B. DUPRÉ, J. VIERS, J.R. NDAM NGOUPAYOU, J.P. BEDIMO BEDIMO, L. SIGHANKAMDJOU, R. FREYDIER, H. ROBAIN, B. NYECK, J. BODIN, P. OLIVA, J.L. BOEGLIN, S. STEMMLER et J. BERTHELIN (2002). Biogeohydrodynamic in the forested humid tropical environment: the case study of the Nsimi small experimental watershed (South Cameroon). Bull. Soc. Geol. Fr., 173, 347-357.

BRGM/OBM (1984). Carte géologique au 1/200 000e, feuille de Djougou-Parakou-Nikki. Office Béninois des Mines, Cotonou, République du Bénin.

CASENAVE A. et C. VALENTIN (1992). A runoff capability classification system based on surface features criteria in semi-arid areas of West Africa. J. Hydrol., 130, 231-249.

CHEVALLIER P. (1990). Complexité hydrologique du petit basin versant. Exemple en savane humide, Booro Borotou (Côte d'Ivoire). ORSTOM Études et Thèses, Paris, 332 p.

CHEVALLIER P. et O. PLANCHON (1993). Hydrological processes in a small humid savanna basin (Ivory Coast). J. Hydrol., 151, 173-191.

CROUZET E., P. HUBERT, P. OLIVE et E.S.A. MARCÉ (1970). Le tritium dans les mesures hydrologiques de surface. Détermination expérimentale du coefficient de ruissellement. J. Hydrol., 11, 217-229.

DANSGAARD W. (1964). Stable isotopes in precipitation. Tellus, 16, 436-468.

DESCLOITRES M., M. WUBDA et Y. LE TROQUER 
(2003). Prospections géophysiques sur le bassin versant d'Ara: électrique $2 D$ et électromagnétisme EM34, compte rendu de mission du 5 au 14 mai 2003, Rapport technique IRD Ouagadougou, Burkina-Faso, 15 p.

FAO (1998). Crop Evaporation - Guidelines for computing crop water requirements. Irrig. Drain. Pap., Rome, 56. http://www.fao.org/docrep/X0490E/X0490E00.htm (consultation le 08 novembre 2006).

FAURE P. et B. VOLKOFF (1998). Some factors affecting regional differentiation of the soils in the Republic of Benin (West Africa), Catena, 32, 281-306.

FAVREAU G. (2000). Caractérisation et modélisation d'une nappe phréatique en hausse au sahel: dynamique et géochimie de la dépression piézométrique naturelle du kori de Dantiandou (sud-ouest du Niger). Thèse de doctorat, Université Paris XI, France, $258 \mathrm{p}$.

GREMILLON P., A. GONYEAU et M. WANIELISTA (2000). Application of alternative hydrograph separation models to detect changes in flow in a watershed undergoing urban development. Hydrol. Proc., 14, 1485-1501.

HARRIS J. (2004). Contribution à l'étude du fonctionnement de la végétation au nord Bénin, suivie phénologique de trois couverts végétaux et mesures de LAI des espèces ligneuses dominantes. DESS, Université Créteil, France, 84 p.

HOOPER R.P. et C.A. SHOEMARKER (1986). A comparison of chemical and isotopic hydrograph separation. Water Resour. Res., 22, 1444.

JOERIN C., K.J. BEVEN, I. IORGULESCU et A. MUSY (2002). Uncertainty in hydrograph separations based on geochemical mixing models. J. Hydrol., 255, 90-106.

KAMAGATÉ B., F. JACQUIN et L. SÉGUIS (2003). Mission nivellement - géochimie, haut-bassin de l'Ouémé (Bénin), rapport technique, IRD Montpellier, $9 \mathrm{p}$.

KAMAGATÉ B. (2006). Fonctionnement hydrologique et origine des écoulements sur un bassin versant en milieu tropical de socle au Bénin: bassin versant de la Donga (haute vallée de l'Ouémé). Thèse de doctorat, Université Montpellier II, France, 319 p.

KENDALL C. et J.J. MCDONNELL (1993). Effect of intrastorm isotopic heterogeneities of rainfall, soil water and groundwater on runoff modelling. Dans: Tracers in Hydrology, proceedings of an international symposium held at Yokohama, Japan, 21-23 July 1993. PETERS, N.E., E. HOEHN, C. LEIBUNDGUT, N. TASE et D.E.
WALLING (Éditeurs). Wallingford (Royaume-Uni). IAHS Press, 215, 41-48.

LEBEL T. et T. VISCHEL (2005). Climat et cycle de l'eau en zone tropicale : un problème d'échelle. CR Geosci., 337, 29-38.

L'HÔTE Y., G. MAHÉ, B. SOME et J.P. TRIBOULET (2002). Analysis of a Sahelian annual rainfall index from 1896 to 2000; the drought continues. J. Sci. Hydrol., 47, 563-572.

ROBAIN H. et M. WUBDA (2004). Rapport de mission au Bénin du 12 octobre au 22 novembre 2004, Rapport technique IRD, $31 \mathrm{p}$.

SKLASH M.G. et R.N. FARVOLDEN (1982). The use of environmental isotopes in the study of high-runoff episodes in streams. Dans : Isotope studies Hydrol. Proc. PERRY JR E.C., W.C. MONTGOMERY (Éditeurs), 65. Northern Illinois University press, Dekalb, Illinois, États-Unis.

TARDY Y., V. BUSTILLO et J.L. BOEGLIN (2004). Geochemistry applied to the watershed survey: hydrograph separation, erosion and soil dynamics. A case study: the basin of the Niger River, Africa. Appl. Geochem., 19, 469-518.

WUBDA M. (2003). Reconnaissance géophysique du bassin versant d'Ara (Djougou, Nord Bénin). DESS de Géophysique Appliquée, Université Pierre et Marie Curie, Paris, France, $65 \mathrm{p}$. 